Service social

\title{
Pour une compréhension de la démarche de recherche d'aide des conjoints violents
}

\author{
Daniel Turcotte, Dominique Damant et Jocelyn Lindsay
}

Volume 44, numéro 2, 1995

Visages de la violence

URI : https://id.erudit.org/iderudit/706694ar

DOI : https://doi.org/10.7202/706694ar

Aller au sommaire du numéro

Éditeur(s)

École de service social de l'Université Laval

ISSN

1708-1734 (numérique)

Découvrir la revue

Citer cet article

Turcotte, D., Damant, D. \& Lindsay, J. (1995). Pour une compréhension de la démarche de recherche d'aide des conjoints violents. Service social, 44(2), 91-110. https://doi.org/10.7202/706694ar
Résumé de l'article

Si la nécessité d'intervenir auprès des conjoints violents est un fait largement reconnu cette population demeure très difficile à rejoindre. Les conjoints violents sont généralement réfractaires à l'idée de consulter et, lorsqu'ils le font, leur persistance dans un programme d'aide est faible. Ce texte vise à dégager une meilleure compréhension de la démarche de recherche d'aide des conjoints violents. La première partie discute de facteurs qui favorisent les comportements de demande d'aide ou y font obstacle. Par la suite, des données de recherche tirées des domaines de la santé mentale et de la toxicomanie sont présentées, du fait de leur similitude avec la violence conjugale. Enfin, la dernière partie aborde certaines pistes de réflexion en ce qui a trait à l'intervention auprès des conjoints violents. 


\title{
Pour une compréhension de la démarche de recherche d'aide des conjoints violents
}

\author{
Daniel TURCOTTE \\ École de service social, Université Laval \\ Dominique DAMANT \\ CRI-VIFF, Université Laval \\ Jocelyn LINDSAY \\ École de service social, Université Laval
}

Initialement considérée comme relevant du domaine de la vie privée, la violence conjugale a été reconnue, depuis une quinzaine d'années, comme un problème social et politique nécessitant une attention particulière et des actions énergiques (Lavigne, 1990). En recherche sociale, les premières études sur le sujet se sont intéressées à l'étendue du phénomène et à ses conséquences sur les victimes. Ces études ont évidemment mis en évidence la nécessité de venir en aide aux femmes victimes de violence. Mais comme plusieurs d'entre elles retournent vivre auprès de leur conjoint après un séjour en milieu d'hébergement et que, s'il y a séparation, la violence du conjoint se porte non seulement sur son ex-femme, mais sur sa nouvelle 
conjointe, l'importance de mettre en place des programmes pour les conjoints violents a également été rapidement reconnue (Lindsay, Ouellet et Saint-Jacques, 1991).

Cependant, les conjoints violents sont généralement réfractaires à l'idée de consulter et, lorsqu'ils le font, leur persistance dans un programme d'aide est généralement faible (Lindsay et al., 1991). Cette situation est d'autant plus préoccupante que le taux élevé d'abandons pose non seulement de sérieux questionnements sur les stratégies à mettre en place pour intervenir efficacement auprès des conjoints violents, mais il contribue à éroder la confiance et la motivation des personnes œuvrant à l'intérieur de cette problématique. Ce texte vise à dégager une meilleure compréhension des comportements de demande d'aide des conjoints violents. La première partie aborde d'une façon générale le processus de recherche $\mathrm{d}^{\prime}$ aide et présente certains facteurs qui favorisent les comportements de demande d'aide ou au contraire y font obstacle. Par la suite, des résultats de recherche sur la sollicitation de l'aide professionnelle dans les domaines de la santé mentale et de la toxicomanie sont exposés. Ces problématiques ont été considérées en raison de leur similitude avec la violence conjugale. Enfin, dans la dernière partie, nous présentons certaines pistes de réflexion en ce qui a trait à l'aide aux conjoints violents.

\section{LE PROCESSUS DE RECHERCHE D'AIDE}

Jusqu'à la fin des années 70, la majorité des recherches sur l'aide professionnelle ont porté sur le pôle «aidant » de la relation d'aide et mettaient l'accent sur les variables qui facilitent ou gênent I'action des aidants (Gross et McMullen, 1983). Depuis, les chercheurs se sont intéressés davantage à la position de la personne aidée en essayant de clarifier le processus de recherche d'aide et de cerner les raisons qui amènent une personne à demander ou à éviter de demander de l'aide lorsqu'elle fait face à une difficulté. Le sujet est d'autant plus préoccupant qu'il est largement reconnu que seule une minorité des personnes qui font face à des problèmes psychosociaux importants recourent à l'aide professionnelle. En fait, la demande d'aide s'inscrit dans un processus complexe qui comporte plusieurs étapes et qui implique de multiples prises de décision (Wills, 1983).

Plusieurs modèles conceptuels ont été élaborés pour décrire la démarche d'une personne qui fait une demande d'aide, que ce soit en santé (Alonzo, 1980 ; Igun, 1979 ; Safer et al., 1979; Suchman, 1965, 
cités dans Gross et McMullen, 1983; Koman, 1993), en santé mentale (Albers et Scrivner, 1977; Gurin, 1960; Kadushin, 1959, cités dans Gross et McMullen, 1983; Horwitz, 1984), en organisation du travail (Shapiro, 1984), en éducation (Dunst et Trivette, 1988) ou en travail social (Landy, 1960). Le modèle le plus souvent mentionné dans les écrits sur le sujet est celui de Gross et McMullen (1983). Selon ce modèle, le processus qui conduit à une demande d'aide se décompose en trois étapes qui, bien que distinctes sur le plan de l'analyse, sont interreliées dans la réalité :

1. la perception de la situation comme un problème pouvant profiter d'une aide extérieure;

2. la décision d'accepter le problème, de le régler seul ou de solliciter de l'aide;

3. Ia mise en place de stratégies permettant d'obtenir l'aide espérée.

\section{LA RECONNAISSANCE DU PROBLÈME}

En ce qui concerne la première étape, des recherches empiriques montrent que des caractéristiques telles que le genre, la classe sociale et la race interviennent dans la reconnaissance d'une situation comme étant problématique. Ainsi, selon Kessler, Brown et Broman (1981), I'explication de la surreprésentation des femmes dans les statistiques sur la maladie mentale se situerait à ce niveau. Comme les femmes ont plus de facilité à voir un symptôme comme un problème nécessitant de l'aide, elles sont plus nombreuses à recourir aux services. D'autres facteurs viennent également influencer l'évaluation que fait une personne de sa réalité, notamment ses expériences antérieures, les normes du milieu, le jugement des autres et la comparaison sociale. Ainsi, l'attitude des personnes présentes dans I'environnement influence la perception d'un symptôme comme étant problématique; certains environnements tendent à nier le problème, voire à le maintenir (Bilodeau, 1994). La comparaison sociale joue aussi un rôle important dans la reconnaissance d'un problème, puisque les personnes en difficulté ont tendance à se situer par rapport à d'autres qui s'en tirent moins bien, évitant ainsi les comparaisons désavantageuses. Par ailleurs, s'attribuer une part de responsabilité dans l'existence d'un problème est une décision encore plus difficile lorsque les difficultés qui sont présentes impliquent plus d'une personne. Dans une telle situation, il est difficile pour la personne de statuer si le problème relève de sa responsabilité, s'il est 
attribuable à quelqu'un d'autre ou s'il résulte de la dynamique de la relation (Wills, 1983).

\section{LA DÉCISION QUANT AU CHOIX DE MESURES}

Une fois le problème reconnu, différentes options se présentent. La personne peut choisir de s'adapter à la situation ou elle peut essayer de s'en sortir par ses propres moyens. Elle peut également voir la situation comme insoluble, donc hors de contrôle ou, encore, elle peut en attribuer la responsabilité à quelqu'un d'autre et se dégager de toute responsabilité. De multiples facteurs peuvent influencer la décision d'une personne quant à l'opportunité de faire appel à une aide extérieure. Les recherches sur le sujet abordent ces facteurs selon deux perspectives: psychologique et sociologique. La première met l'accent sur les caractéristiques de la personne, alors que la seconde s'intéresse davantage aux interactions sociales qui ont cours dans le processus d'aide.

\section{L'influence des caractéristiques personnelles}

Dans une recension des écrits sur les caractéristiques personnelles qui influencent la décision de solliciter de l'aide, Nadler (1983) s'est particulièrement intéressé à l'estime de soi. À ce niveau, deux hypothèses s'affrontent. Selon la première, les individus ayant une faible estime de soi hésiteront davantage à consulter car ils doivent se protéger; pour eux, demander de l'aide constitue une menace à l'estime de soi. Selon la deuxième hypothèse, ce sont au contraire les personnes avec une haute estime de soi qui seront plus réfractaires à consulter. Ces personnes se sentent menacées par l'idée de demander de l'aide, car cette démarche vient remettre en question l'image de compétence qu'elles ont d'elles-mêmes. Les recherches empiriques vont dans le sens de cette deuxième hypothèse: les personnes ayant une haute estime d'elles-mêmes sont généralement plus réfractaires à rechercher de l'aide par leur désir d'éviter d'expérimenter une dissonance entre leurs standards internes et l'inadéquation associée au fait de demander de l'aide (Nadler, 1983).

La propension à solliciter de l'aide serait également influencée par la façon dont la personne explique ses problèmes. S'appuyant sur la théorie de I'attribution, plusieurs recherches ont établi que les personnes sont moins portées à demander de l'aide lorsqu'elles attribuent leurs difficultés à leurs limites personnelles plutôt qu'à des 
facteurs externes: "Si le besoin d'aide est attribué à l'inadéquation personnelle, la personne développe une image d'elle-même moins positive et elle a moins tendance à demander de I'assistance que si le besoin est attribué à des facteurs extérieurs » (Fisher, Nadler et Withner-Alagna, 1983: 62).

D'autres recherches ont fait ressortir que des variables comme le site de contrôle (locus of control) et la capacité de parler de soi interviennent également dans le processus de demande d'aide. Ainsi, plus une personne a un foyer de contrôle interne, plus elle démontrera une attitude positive à l'égard de la recherche d'aide (Tijhuis, Peters et Foets, 1990). Par ailleurs, la difficulté à se dévoiler et à parler de soi a été associée à une faible propension à demander de l'aide (Good, Dell et Mintz, 1989; Fisher, Winer et Abramowitz, 1983).

Les recherches sur l'appartenance socioculturelle mettent en évidence le lien entre les variables de genre, de classe sociale et de race, et la facilité ou la difficulté à faire une demande d'aide. La plupart des recherches montrent que les hommes ont plus de difficulté à reconnaître un problème et à demander de l'aide (Guberman et al., 1993). Certaines recherches révèlent que les personnes ayant un statut socio-économique plus faible sont moins susceptibles de faire appel à l'aide institutionnelle ou formelle (Williams et Williams, 1983). Par ailleurs, lorsqu'elles font une telle demande, elles ont tendance à adopter un style didactique, c'est-à-dire à demander des instructions précises sur la façon de résoudre leur problème, alors que les personnes de milieu aisé favorisent plus souvent un style de négociation: I'aide demandée est vue comme un élément visant à faciliter leur démarche de résolution de problème (Asser, 1978).

\section{La perspective sociologique}

Les recherches qui ont abordé le comportement de demande d'aide sous l'angle des interactions sociales s'appuient sur deux théories: la théorie de l'échange et la théorie de l'obligation (indebtedness theory). Selon la théorie de l'échange, les interactions humaines sont une suite de transactions qui comportent des coûts et des bénéfices. Comme chaque individu a intérêt à minimiser ses coûts et à maximiser ses bénéfices, il aura tendance à éviter les situations et les comportements coûteux et à rechercher les statuts et les interactions qui lui procurent des bénéfices. Ainsi, selon la théorie de l'échange, la capacité d'une personne à développer et à maintenir un réseau de relations soutenantes dépendra de son habileté à donner aussi bien qu'à recevoir (Antonucci et Jackson, 1990; Vaux, 1988). 
Dans la même perspective, la théorie de l'obligation souligne que le fait de recevoir un bénéfice crée un état d'obligation qui exige une forme de remboursement (Greenberg, 1980; Greenberg et Westcott, 1983). Dans une dynamique d'aide, ce sentiment d'obligation est influencé par la perception qu'a la personne aidée: (1) de la motivation de I'aidant, (2) de l'importance des bénéfices et des coûts de l'échange pour chacune des parties, (3) de l'attribution de la responsabilité de l'action de l'aidant et (4) de la similitude de sa situation par rapport à celle d'autres personnes dans le même cas (Greenberg, 1980). Si les personnes en difficulté sont plus susceptibles de demander de l'aide lorsqu'elles estiment que l'aidant subit peu d'inconvénients et récolte des bénéfices importants de son action, elles ont, par contre, un sentiment d'obligation plus marqué lorsqu'elles sollicitent elles-mêmes cette aide (Gross et McMullen, 1983). Toutefois, I'obligation de réciprocité prendra une teinte différente selon la nature de la relation entre les parties concernées. Ainsi, selon Wentewski (1981 cité dans Antonucci et Jackson, 1983), lorsqu'une relation est plutôt superficielle, les règles de l'échange sont strictes: la réciprocité est immédiate et de valeur équivalente. Par contre, les relations intimes et continues dans le temps ont tendance à exiger un retour moins immédiat et sans équivalence parfaite. Cette observation rejoint la distinction de Clark (1983) entre les relations communales et les relations d'échange. Dans ce dernier type de relations, les membres retournent rapidement un bénéfice comparable à celui reçu, signifiant ainsi clairement que la dette a été remboursée. Dans les relations communales, la nature du bénéfice est fonction des besoins de l'autre plutôt que semblable au bénéfice reçu, et le retour se fait lorsque I'occasion se présente. Antonucci et Jackson (1990) parlent de banque de soutien pour expliquer le fait que les échanges sociaux avec les proches n'entraînent pas une réciprocité immédiate.

Ces données sur les facteurs qui influencent la décision de solliciter ou non de l'aide mettent en lumière le fait qu'une personne sera moins susceptible de faire appel à des ressources extérieures si elle se sent menacée dans son estime de soi ou dans sa liberté, si elle interprète sa demande comme un signe de défaillance personnelle et si elle s'interroge sur ses capacités de remplir ses obligations en matière de réciprocité. En outre, elles permettent de croire que la personne sera plus susceptible de faire appel aux ressources qu'elle perçoit comme moins menaçantes, moins culpabilisantes et moins exigeantes sur le plan de la réciprocité. 


\section{LE CHOIX DE LA MESURE D'AIDE}

La troisième étape du processus de demande d'aide porte sur le choix d'une ressource d'aide appropriée. À cet égard, les résultats de recherche indiquent que la sollicitation de l'aide auprès des professionnels survient habituellement après des démarches auprès d'amis et de membres de la famille (Crespo-Medina, 1988; Gourash, 1978; Bennet-Veroff, 1981, cité dans Wills, 1992). Ces sources d'aide informelle correspondent au réseau de soutien social d'une personne.

\section{Le soutien social comme source d'aide}

Le soutien social correspond à l'ensemble des fonctions aidantes qui sont réalisées par les personnes significatives de l'entourage d'une personne. Il ne correspond pas à une caractéristique personnelle statique ou à une donnée de l'environnement; il se présente plutôt comme le résultat de transactions complexes entre la personne et son environnement. La représentation du soutien passe par l'appréciation de trois composantes: les ressources de soutien, les comportements supportants et l'appréciation subjective du soutien (Hobfoll et Vaux, 1993 ; Letham et Duck, 1990 ; Sarason, Sarason et Pierce, 1990; Vaux, 1988).

Les fonctions attribuées au soutien social sont multiples et elles font l'objet de classifications fort variées. Parmi ces fonctions, on retrouve, notamment, l'acquisition d'un sentiment d'identité, l'encouragement et le feed-back positif, la protection contre le stress, le développement des connaissances, habiletés et ressources, I'aide matérielle ou financière, I'acquisition de l'estime de soi, la socialisation et la validation personnelle (Cameron, 1990 ; Cutrona et Russell, 1990; Vaux, 1988). Pour articuler l'ensemble de ces fonctions, Cutrona et Russell (1990) proposent une classification à cinq composantes :

1. le soutien émotionnel, qui consiste en la possibilité d'en appeler à d'autres pour être sécurisé et réconforté dans les périodes de stress;

2. le soutien «informationnel», qui est la provision d'informations ou de conseils permettant de composer efficacement avec les problèmes quotidiens ;

3. I'aide concrète, c'est-à-dire l'assistance instrumentale tangible;

4. le soutien de l'estime de soi, qui correspond à l'enrichissement par les autres du sentiment de compétence et de valeur personnelle; 
5. I'intégration sociale, soit le sentiment de faire partie d'un groupe qui partage des préoccupations et des intérêts communs.

Cette typologie met en présence deux types de fonctions: la gestion des difficultés et le développement personnel. Ainsi, le soutien émotionnel, l'échange d'information et l'aide concrète font référence à des éléments qui permettent de faire face aux difficultés qui se présentent. L'intégration sociale et le soutien à l'estime de soi favorisent la validation personnelle et l'acquisition d'un sentiment d'appartenance; ils sont davantage reliés à l'identité sociale et au bien-être psychologique qu'à la résolution de problèmes précis. Ces deux types de fonctions correspondent aux deux modèles dominants dans l'étude des effets du soutien social: I'effet tampon et l'effet direct. Le modèle de l'effet tampon stipule que l'influence négative d'un événement stressant sera atténuée si une personne bénéficie d'un soutien social adéquat. Le soutien social protège des effets négatifs du stress sur la santé et le bien-être psychologique (DunkelSchetter et Bennett, 1990; Vaux, 1988). Selon le modèle de I'effet direct, le soutien social a un effet sur le bien-être des personnes, indépendamment des sources de stress, puisqu'il offre la possibilité de vivre des expériences positives à travers un réseau stable de relations. Donc, si le soutien social est un facteur qui contribue au développement d'une personne, son impact est encore plus significatif lorsqu'une personne fait face à des difficultés majeures.

Bien que la littérature sur le soutien social ait connu une croissance considérable aux cours des deux dernières décennies, la plupart des recherches ont porté sur la nature et les effets du soutien social (Burleson, 1990). Peu d'attention a été accordée à la signification des interactions qui ont cours entre la personne soutenue et la personne soutenante (Eckenrode et Wethington, 1990; Hobfoll et Vaux, 1993; Sarason, Sarason et Pierce, 1990) et à l'étude des motifs qui font que certaines personnes ne demandent pas l'aide dont elles ont besoin lorsque celle-ci est disponible (Gross et McMullen, 1983). Pour aborder cette question, il apparaît utile, dans un premier temps, de clarifier la notion de mobilisation du soutien, notion étroitement associée à la demande d'aide.

Selon Eckenrode et Wethington (1990), la mobilisation du soutien correspond à un processus de rassemblement de ressources en anticipation ou en réponse à une menace ou une difficulté. Cette mobilisation se réalise à travers deux modalités: I'offre et la sollicitation du soutien. Ces deux modalités se distinguent sur plusieurs aspects. Ainsi, le soutien sollicité résulte généralement d'une expression verbale des problèmes vécus ou d'une demande explicite d'aide, 
alors que l'offre de soutien émerge spontanément. En outre, l'offre de soutien s'exprime plus facilement face à des difficultés visibles et non stigmatisantes, alors que la sollicitation est souvent nécessaire lorsque la situation est particulièrement problématique ou lorsque le réseau de soutien est inadéquat (Moos et Mitchell, 1982). La sollicitation du soutien, ou la demande d'aide, qui est une modalité mise en branle lorsque le soutien offert ne suffit pas à répondre aux besoins, est une démarche qui comporte certains risques. L'embarras (Shapiro, 1984), la peur de voir sa demande refusée (DePaulo, 1982), I'inconfort à révéler ses limites (Rosen, 1983), le désir de ne pas se créer d'obligation (Greenberg et Westcott, 1983) et la volonté de réussir par soi-même (Ames, 1983) sont autant de facteurs qui peuvent freiner l'élan d'une personne à solliciter de I'aide.

De la même façon que la sollicitation du soutien est utilisée pour compenser l'insuffisance de l'aide offerte spontanément, la demande $d^{\prime}$ aide auprès d'une ressource formelle survient le plus souvent lorsque le soutien provenant du réseau social est insuffisant ou inadéquat. En ce sens, le soutien social influence le processus de demande d'aide de différentes façons. Il peut: a) servir de tampon en faisant diminuer le stress et par conséquent le besoin d'aide, b) procurer le soutien instrumental et affectif nécessaire et par le fait même éliminer le besoin d'aide, c) favoriser l'orientation auprès des services professionnels et d) contribuer à transmettre des attitudes, des valeurs et des normes au sujet du fait de demander de l'aide (Gourash, 1978). Le réseau social exerce donc une fonction de filtrage et de référence qui influence la recherche d'aide auprès des ressources formelles (Wills, 1992). En fait, I'utilisation des ressources d'aide peut s'inscrire dans un cycle récurrent où "I'utilisation d'une ressource informelle favorise le recours à une autre ressource formelle, de telle sorte que les mêmes personnes reçoivent davantage d'aide et bénéficient d'une aide plus diversifiée »(Cantin, Rinfret-Raynor et Fortin, $1994:$ 77).

\section{La recherche d'aide auprès des ressources formelles: quelques comparaisons}

La littérature sur I'utilisation des ressources formelles d'aide est souvent présentée en relation avec les problèmes vécus par les personnes qui font appel aux services, puisque les ressources formelles sont généralement spécialisées et qu'elles visent des clientèles particulières. Or, dans le domaine de la violence conjugale, aucune recherche portant spécifiquement sur la sollicitation de l'aide auprès 
des ressources formelles par les conjoints violents n'a pu être relevée. Nous avons donc procédé à une exploration sommaire des recherches sur le sujet dans deux problématiques connexes, soit la santé mentale et la toxicomanie.

L'écart entre les hommes et les femmes sur le plan des diagnostics et des traitements en santé mentale est un phénomène bien connu (Tousignant, 1987). D'une part, les diagnostics attribués aux hommes et aux femmes diffèrent (Dorvil, 1985). Alors que l'alcoolisme, les troubles de la personnalité, les désordres sexuels et la dépendance aux drogues sont principalement des symptômes masculins, les névroses dépressives et la détresse psychologique sont des symptômes surtout féminins (Corbeil, Pâquet-Deehy, Lazure et Legault, 1983; Guberman et al., 1993). D'autre part, les femmes consultent davantage: elles éprouvent des sentiments plus positifs à l'égard du soutien social et sont plus portées à se tourner vers I'aide professionnelle quand le réseau informel n'est plus efficace (Bennet-Veroff, 1981).

Selon Fischer, Miner et Abramowitz (1983), cette situation s'explique par le fait que les femmes ont une attitude plus favorable à l'égard de la demande d'aide, attitude qui, de l'avis de McMullen et Gross (1983), relèverait en large partie de la socialisation. En effet, des qualités comme la compétence, l'autosuffisance, I'indépendance sont autant de traits considérés comme masculins, alors qu'avoir besoin d'aide est plus facilement associé à une caractéristique féminine. Par ailleurs, demander de I'aide implique une forme de dépendance à l'égard de l'aidant, ce qui vient en contradiction avec la valorisation de l'autonomie faite auprès des garçons. En outre, les hommes ont tendance à moins dévoiler leur intimité et leurs caractéristiques négatives.

Dans son étude sur l'influence du genre sur les attitudes à l'égard de la recherche d'aide, Johnson (1988) ne relève aucune différence entre les hommes et les femmes sur le plan de la confiance vis-à-vis des services d'aide. Par contre, les femmes sont plus tolérantes au stigmate que la demande d'aide peut produire et elles sont plus ouvertes au dévoilement d'un problème personnel. Par ailleurs, chez les hommes, les sujets classés comme étant d'orientation plus féminine ont démontré plus de confiance envers les professionnels. S'appuyant sur I'utilisation de deux questionnaires portant sur les attributs personnels, dans un cas (Extended Personal Attribute Questionnaire), et sur I'utilisation du soutien social, dans I'autre (Natural Support Questionnaire), Butler, Giordano et Neren (1985) ont fait ressortir que les hommes ayant des attributs associés à des traits féminins amorcent plus facilement une demande d'aide. 
Good, Dell et Mintz (1989) ont tenté de mesurer l'influence de trois éléments sur le processus de demande d'aide, soit la difficulté d'exprimer ses émotions, le système de valeurs des individus et la peur de l'intimité et du rapprochement émotionnel. Les résultats de leur étude menée auprès de 401 étudiantes et étudiants indiquent que les attitudes traditionnelles au sujet du rôle masculin (expression des émotions et de l'affection) étaient associées à des attitudes négatives à l'égard d'une demande d'aide psychologique. Dans le même sens, Robertson (1989) s'est intéressé aux liens entre les conflits de genre, les attitudes stéréotypées et les attitudes à l'égard du fait de demander de l'aide. Il a trouvé que les hommes avec des scores élevés sur les sous-échelles de succès et d'émotivité restreinte avaient les attitudes les plus négatives vis-à-vis de l'aide professionnelle. Les hommes d'orientation féminine et ayant des scores moins élevés dans les sous-échelles pouvoir et émotivité restreinte étaient plus favorables à la thérapie. Partant de ces observations, il s'est demandé si la représentation de l'aide psychosociale ne constituait pas en soi un obstacle à l'engagement des hommes dans la mesure où l'image généralement répandue de cette forme d'aide apparaît comme antinomique à des traits typiquement masculins comme le succès, la compétition, le pouvoir. Ainsi, il a émis l'hypothèse que la façon de présenter l'aide pouvait influencer la propension des hommes à y avoir recours. Pour vérifier son hypothèse, il a construit deux brochures: I'une décrivant de manière classique des services de thérapie plus traditionnels et une autre décrivant dans des mots plus conformes aux stéréotypes masculins des services moins traditionnels. Cette dernière brochure parlait en termes de résolution de problèmes et offrait des cours, séminaires et ateliers plutôt que de la thérapie. Après analyse, il a relevé que les hommes aux traits très masculins préféraient cette dernière brochure, qui ne rebutait pas pour autant les hommes d'orientation plus féminine.

L'ensemble de ces travaux de recherche indique qu'il existe une différence entre les hommes et les femmes sur le plan des comportements de demande d'aide dans le domaine de la santé mentale: les hommes sont non seulement moins enclins à reconnaître leurs problèmes, mais ils sont plus réfractaires à demander de l'aide. Cette tendance est cependant moins marquée chez les hommes qui ont une vision moins stéréotypée du rôle masculin.

La problématique de l'alcoolisme et de la toxicomanie présente des similitudes évidentes avec celle de la violence conjugale: les personnes confrontées à cette difficulté prennent généralement beaucoup de temps à reconnaître leur problème et à demander de l'aide 
et leur démarche résulte le plus souvent des pressions de leur entourage. En outre, les hommes violents sont largement représentés chez les personnes alcooliques. En effet, selon la recension de Tolman et Bennett (1990), la moitié des « abuseurs » en traitement ont également des problèmes d'alcoolisme ou de toxicomanie. Il apparaît donc intéressant d'explorer sommairement les écrits sur les comportements de recherche d'aide des personnes alcooliques.

Sur le plan de la reconnaissance du problème, les écrits révèlent que si le volume d'alcool ingurgité quotidiennement est souvent l'indice qui fait reconnaître l'existence d'un problème d'alcoolisme, la décision de demander de l'aide se présente généralement comme la résultante de plusieurs événements précipitants (problèmes relationnels, problèmes physiques, problèmes avec la loi) (Jordan et Oei, 1989). Si la peur d'avoir perdu le contrôle sur sa consommation peut être un facteur précipitant la demande d'aide chez les alcooliques (Brooke, Fudala et Johnson, 1992), c'est souvent à partir du moment où elle interfère dans la performance des rôles au travail ou au sein de la famille que la consommation est reconnue comme un problème (Thom, 1986).

La reconnaissance du problème ne conduit pas nécessairement à une recherche d'aide, puisque la peur du traitement, la gêne devant l'éventualité de ne pas être vue comme une personne normale et respectable, la peur de l'échec et la crainte de décevoir les autres constituent autant de barrières à un engagement dans une démarche d'aide (Brooke et al., 1992). Même une fois engagé dans un processus d'aide, il demeure difficile de modifier les comportements de consommation, notamment à cause de l'influence du réseau social. La recherche d'aide chez les personnes alcooliques apparaît donc comme une démarche qui est stimulée par des facteurs externes (problèmes relationnels, troubles de santé, dispositions légales), qui est freinée par des facteurs internes (gêne, peur de l'échec, crainte de décevoir) et dont les résultats sont soumis à diverses influences externes.

\section{CARACTÉRISTIQUES PERSONNELLES DES HOMMES VIOLENTS ET RECHERCHE D'AIDE}

Pour mieux situer la portée de ces données au regard de la réalité des hommes violents, rappelons d'abord les principaux traits qui sont associés à ces personnes. La recension d'écrits de Ouellet, Lindsay et Saint-Jacques (1993) fait ressortir que les conjoints violents: 
- nient ou minimisent leur violence,

- sont dépendants et jaloux,

- ont une faible estime de soi,

- éprouvent des problèmes de contrôle de leur impulsivité,

- manquent de respect à l'égard des normes sociales,

- souffrent d'acting-out antisocial chronique,

- vivent un stress extrême quand ils ont perdu le contrôle de leur vie,

- ont une forte tendance à différentes dépendances,

- vivent des dépressions situationnelles,

- ont une conception rigide et stéréotypée des rôles de l'homme et de la femme,

- ont fréquemment été victimes ou témoins de violence pendant leur enfance.

De plus, si certains sont dépressifs, colériques et jaloux, d'autres manifestent une violence généralisée et sévère associée à des problèmes d'alcool (Saunders, 1990). En fait, les hommes qui sont violents exclusivement avec leur conjointe ont une bonne estime de soi, ne sont pas dépressifs et sont moins violents que les hommes qui ont une violence généralisée. Ces derniers ont des relations maritales moins durables et de plus courte durée et leur violence est plus fréquente et plus sévère (Cadsky et Crawford, 1988).

En combinant les données de recherche sur les facteurs qui contribuent ou font obstacle à la recherche d'aide avec les caractéristiques des conjoints violents, on ne s'étonne pas qu'ils soient particulièrement difficiles à rejoindre. En effet, ils présentent plusieurs caractéristiques qui sont considérées comme des barrières à l'insertion dans un processus d'aide : négation de leur problème, site de contrôle externe, conception stéréotypée des rôles de l'homme et de la femme. En outre, ils attendent souvent des événements précipitants venant de l'extérieur pour faire une demande d'aide, ils ont un problème jugé socialement inacceptable et ils ont souvent des amis ou un entourage qui nient ou encouragent des attitudes de violence.

Malgré ce profil, certains s'engagent dans une démarche d'aide. Selon l'étude de Rondeau (1989), les principales motivations à consulter sont la peur de perdre la conjointe et les conséquences légales. Cependant, le taux d'abandon est très élevé (Piros-Good et Stets-Kealy, 1985). Les hommes qui terminent le traitement sont généralement mieux éduqués, occupent un emploi, ont un plus grand 
nombre d'enfants et ils ont été témoins de violence plutôt que victimes eux-mêmes (Carrillo, 1985; Grusznski et Carrillo, 1988; Saunders et Parker, 1989; Syers et Edleson, 1992). Enfin, des recherches sur l'effet différentiel de l'arrestation sur les nouveaux incidents de violence tendent à montrer une influence de l'emploi et du statut marital sur la récidive (Berk, Campbell, Klap et Western, 1992; Sherman et al., 1992 ; Syers et Edleson, 1992). Toutefois, pour Berk et al. (1992), ces deux facteurs sont liés à un ensemble d'autres variables psychologiques et sociales.

\section{CONCLUSION}

Ce texte a relevé certains éléments qui influencent les comportements de demande d'aide chez les personnes en difficulté. À partir d'une définition de la démarche de demande d'aide comme un processus comportant trois étapes, différents facteurs susceptibles d'être associés aux comportements des personnes à chacune de ces étapes ont été relevés dans l'optique d'en arriver à une meilleure compréhension de la réticence des conjoints violents à s'engager et à persévérer dans une démarche d'aide. Il en ressort que plusieurs des caractéristiques qui se retrouvent chez les conjoints violents sont définies dans la littérature comme des éléments qui freinent la propension à amorcer une démarche de recherche d'aide. II n'est donc pas étonnant que ces personnes soient particulièrement difficiles à rejoindre. Par contre, il est possible de dégager certaines pistes qui peuvent modifier cette situation.

Ainsi, sur le plan de la reconnaissance de la violence comme un problème, il est nécessaire de poursuivre les actions actuelles de dénonciation de la violence afin de faire en sorte que les normes sociales conduisent rapidement à la reconnaissance de la violence, quelle qu'en soit la forme, comme étant un comportement problématique qui doit appeler à des mesures d'aide. Cette dénonciation devrait porter sur tous les niveaux de violence et ne pas se limiter aux situations extrêmes. En effet, il faut éviter que les conjoints violents minimisent leur propre violence en se comparant avec des personnes qui ont des comportements pires que les leurs. Cette précaution est d'autant plus importante que les conjoints violents se retrouvent souvent dans un environnement qui valorise le recours à la violence comme modalité de résolution des conflits.

En ce qui a trait à la décision de demander de l'aide, il serait essentiel de présenter le recours à une mesure d'aide comme un geste 
positif. Si la violence doit être dénoncée, l'attitude du conjoint qui décide de s'engager dans une démarche d'aide doit être valorisée et renforcée. Par ailleurs, il faut que cette initiative conduise à des bénéfices qui l'emportent sur les inconvénients que comporte le fait de participer à une intervention. En ce sens, il est nécessaire, d'une part, de cerner rapidement les événements qui sont à l'origine de la demande d'aide et, d'autre part, de percevoir les coûts associés à I'abandon des comportements de violence. II ne faut pas sous-estimer les obstacles qui se dressent devant le changement ni présumer que toute modification de comportement dans la direction visée par I'intervention comporte nécessairement des avantages à court terme pour la personne.

$S^{\prime}$ il est nécessaire de faire connaître davantage les services qui sont disponibles pour les conjoints violents, il est également indispensable de faire en sorte que l'image de ces services qui est véhiculée soit attrayante pour ces personnes. Il faut aussi viser à ce que les approches utilisées permettent, tant par leur processus que par leurs résultats, de maintenir, voire d'augmenter, la motivation des conjoints qui entreprennent une demande d'aide. Il y aurait également avantage à agir sur le milieu environnant pour que son influence soutienne le changement plutôt que normalise la violence. En complémentarité avec ces actions, il est nécessaire de poursuivre la recherche sur les comportements de demande d'aide des conjoints violents, afin de mieux comprendre la logique de la démarche des conjoints qui s'engagent dans un processus d'aide. II serait utile, notamment, de mieux connaître les facteurs à l'origine de cette démarche, de cerner les conditions qui facilitent le maintien de l'engagement dans le processus d'intervention ou celles qui font obstacle et de dégager des éléments qui sont associés aux résultats.

L'intervention auprès des conjoints violents est une réalité complexe qui touche plusieurs dimensions de la vie des personnes concernées et qui implique plusieurs types d'acteurs. Devant une telle complexité, les solutions toutes faites et les réponses expéditives sont généralement non seulement inefficaces, mais contreproductives. C'est plutôt à travers une remise en question permanente de ses façons de faire et par une collaboration étroite entre intervenants et chercheurs que sont susceptibles d'émerger des nouvelles avenues d'intervention plus en mesure de répondre à ce problème incontournable qu'est la violence et, plus spécifiquement, la violence familiale. 


\section{Références bibliographiques}

AMES, R. (1983). "Help-seeking and achievement orientations : perspective from attribution theory ", dans B.M. DePaulo, A.J. Nadler et J.D. Fischer (dir.), New directions in helping. Help-seeking, p. 165-186. New York: Academic Press.

ANTONUCCI, T.C. et J.S. JACKSON (1990). "The role of reciprocity in social support», dans B.R. Sarason, I.G. Sarason et G.R. Pierce (dir.), Social support: an interactional view, p. 173-198. New York: John Wiley \& Sons.

ASSER, E.S. (1978). Social class and help-seeking behavior, American Journal of Community Psychology, vol. 6 : 465-494.

BenNeT-VEROFF, J. (1981). "The dynamics of help-seeking in men and women: a national survey », Psychiatry, vol. 44 : 189-200.

Berk, R., A. Campbell, R. Klap et B. Western (1992). "The deterrent effect of arrest in incidents of domestic violence: a bayesian of four field experiments », American Sociological Review, vol. 57 :698-708.

BilodeAu, A. (1994). "Dynamique de la recherche d'aide des femmes victimes de violence conjugale», dans M. Rinfret-Raynor et S. Cantin (dir.). Violence conjugale. Recherches sur la violence faite aux femmes en milieu conjugal, Montréal: Gaëtan Morin Éditeur, 513 pages.

BROOKE, D., P.J. FUdALA et R.E. JOHNSON (1992). "Weighing up the pros and cons: help-seeking and drug misusers in Baltimore, USA », Drug and alcohol dependance, vol. 31:37-43.

BURLESON, B.R. (1990). "Conforting as social support: relational consequences of supportive behaviors », dans S. Duck (dir.), Personal relationships and social support, p. 66-82. Newbury Park: Sage.

Butler, T., S. Giordano et S. Neren (1985). "Gender and sex-role attributes as predictors of utilization of natural support systems during personal stress events », Sex Roles, vol. 13, no 9-10: 515-524.

CADSKY, O. et M. CRAWFORD (1988). «Establishing batterer typologies in a clinical sample of men who assault their female partners », Canadian Journal of Community Mental Health, vol. 7, nº $2:$ 119-127.

CAMERON, G. (1990). "The potential of informal social support strategies in child welfare », dans M. Rothery et G. Cameron (dir.), Child maltreatment: expanding our concept of helping, p. 145-168. Hillsdale, NJ : Lawrence Erlbaum.

CANTIN, S., M. RINFRET-RAYNOR et L. FORTIN (1994). Utilisation des ressources par les victimes de violence conjugale. Le cas des femmes référées aux CLSC par les policiers. Montréal: CRI-VIFF.

CARRILLO, T.P. (1985) A study of men who batter: demographic and psychological indicators for prediction of completion of treatment. University of Minnesota, Doctoral Dissertation.

CLARK, M.S. (1983). "Some implications of close social bonds for helpseeking ", dans B.M. DePaulo, A. Nadler et J.D. Fisher (dir.), New directions in helping. Help-seeking, volume 2, p. 205-230. New York : Academic Press. 
Corbeil, C., C. PÂquet-Deehy, C. Lazure et G. Legault (1983). L'intervention féministe: I'alternative des femmes au sexisme en thérapie. Montréal : Éditions coopératives Albert Saint-Martin.

CRESPO-MEDINA, J.D. (1988). Help-seeking behavior and its social context: social networks, network orientation and social acceptability of the problem. Boston: Boston University, Doctoral Dissertation.

Cutrona, C.E. et D.W. Russell (1990). "Type of social support and specific stress: toward a theory of optimal matching », dans B.R. Sarason, I.G. Sarason et G.R. Pierce (dir.), Social support: an interactional view, p. 319-365. New York: John Wiley \& Sons.

Cutrona, C.E., J.A. SuHR et R. MACFARLANe (1990). "Interpersonal transactions and the psychological sense of support», dans S. Duck (dir.), Personal relationships and social support, p. 30-45. Newbury Park: Sage.

DePaulo, B.M. (1982). "Social-psychological processes in informal helpseeking ", dans T.A. Wills (dir.), Basic processes in helping relations, p. 255-279. New York: Academic Press.

DORVIL, H. (1985). "Types de sociétés et de représentations du normal et du pathologique», dans J. Dufresne, F. Dumont et Y. Martin (dir.), Traité d'anthropologie médicale: l'institution de la santé et de la maladie, p. 305-332. Sainte-Foy: Presses de I'Université du Québec, IQRC et Presses universitaires de Lyon.

Dunkel-SChetter, C. et T.L. Bennett (1990). "Differentiating the cognitive and behavioral aspects of social support», dans B.R. Sarason, I.G. Sarason et G.R. Pierce (dir.), Social support: an interactional view, p. 267-295. New York: John Wiley \& Sons.

Dunst, C.J. et C.M. TRIVETTE (1988). "Helping, helplessness and harm », dans J.C. Witt, S.N. Elliott et F.M. Gresham (dir.), Handbook of behavior therapy in education, p. 343-376. New York: Plenum Press.

ECKENRODE, J. et E. WeTHINGTON (1990). "The process and outcome of mobilizing social support», dans S. Duck et R.C. Silver (dir.), Personal relationships and social support, p. 83-103. Newbury Park: Sage.

FISHER, E.H., D. Winer et S.I. AbRAMOWITZ (1983). "Seeking professional help for psychological problems», dans A. Nadler, J.D. Fisher et B.M. DePaulo, (dir.) New directions in helping. Applied perspectives on help-seeking and receiving, p. 163-185. New York: Academic Press.

GoOd, G.E., D.M. DeLl et L.B. Mintz (1989). "Male role and gender role conflict: relations to help seeking in men», Journal of counseling psychology, vol. 36, $\mathrm{n}^{\circ} 3:$ 295-300.

GOURASH, N. (1978). "Help-seeking: a review of the literature", American journal of community psychology, vol. 6, $\mathrm{n}^{\circ} 5: 413-423$.

GreEnBERG, M.S. (1980). "A theory of indebtedness», dans K. Gergen, M.S. Greenberg et R.H. Willis (dir.), Social exchange: Advances in theory and research. New York: Plenum Press.

GreEnBERG, M.S. et D.R. WestCott (1983). «Indebtedness as a mediator of reactions to aid», dans J.D. Fisher, A. Nadler et B.M. DePaulo (dir.), New directions in helping: Recipient reactions to aid, p. 85-112. New York: Academic Press. 
Gross, A.E. et P.A. MCMulLen (1983). "Models of help-seeking process", dans B.M. DePaulo, A. Nadler et J.D. Fisher (dir.), New directions in helping. Help-seeking, p. 45-70. New York: Academic Press.

GRUSZNSKI, R.J. et T.P. CARRILLO (1988). "Who completes batterer's treatment groups? An empirical investigation », Journal of family violence, vol. 3, $\mathrm{n}^{\circ} 2$ : 141-150.

Guberman, N., J. Broué, J. LindSAy et L. SpeCtOr (1993). Le défi de l'égalité. La santé mentale des hommes et des femmes. Boucherville: Gaëtan Morin Éditeur.

HELlER, K., R.H. PRICE et J.R. HOGG (1990). "The role of social support in community and clinical intervention », dans B.R. Sarason, I.G. Sarason et G.R. Pierce (dir.), Social support: an interactional view, p. 427-453. New York: John Wiley \& Sons.

HOBFOLL, S.E. et A. VAUX (1993). "Social support: social resources and social context», dans L. Golberger et S. Breznitz (dir.), Handbook of stress: theoretical and clinical aspects, p. 685-706. New York: The Free Press.

HORWITZ, A.V. (1987). "Help-seeking processes and mental health services », dans Improving mental health services: what the social sciences can tell us, San Francisco: Jossey-Bass.

JOHNSON, M.E. (1988). "Influences of gender and sex role orientation on help-seeking attitudes», The Journal of Psychology, vol. 122, $\mathrm{n}^{\circ} 3$ : 237-241.

JORDAN, C.M. et T.P.S. OEI (1989). «Help-seeking behaviour in problems drinkers: a review », British Journal of Addiction, vol. 84 : 979-988.

KAPLAN, R.M. et M.T. TOSHIMA (1990). "The functional effects of social relationships on chronic illness and disability », dans B.R. Sarason, I.G. Sarason et G.R. Pierce (dir.), Social support: an interactional view, p. 427-452. New York: John Wiley \& Sons.

KESSLER, R.C., R.L. BROWN et C.L. BROMAN (1981). "Sex differences in psychiatric help-seeking: evidence from four large-scale survey », Journal of health and social behavior, vol. $22: 49-64$.

KOMAN, J.J. III (1993). "Initial report of a new instrument to measure the decision to seek medical treatment », Psychology: A journal of human behavior, vol. 30, $\mathrm{n}^{\circ} 1: 35-39$.

LANDY, D. (1965). "Problems of the person seeking help in our culture», dans M.N. Zald (dir.), Social welfare institutions, p. 559-574. New York: John Wiley \& Sons.

LAVIGNe, M. (1990). "D'une décennie de femmes à l'autre», Perception, vol. $14, \mathrm{n}^{\circ} 2: 50-52$.

LeAtham, G. et S. DuCK (1990). "Conversations with friends and the dynamics of social support », dans S. Duck (dir.), Personal relationships and social support, p. 1-29. Newbury Park: Sage.

LINDSAY, J., F. OUELLET et M.-C. SAINT-JACQUES (1991). Les groupes de traitement pour conjoints violents : recension critique portant sur le traitement, son efficacité, sa mesure. Sainte-Foy: Centre de recherche sur les services communautaires, Université Laval. 
MCMullen, P.A. et A.E. Gross (1983). "Sex differences, sex roles and health-related help-seeking», dans B.M. DePaulo, A. Nadler, J.D. Fisher (dir.), New directions in helping. Help-seeking, p. 233-263. New York: Academic Press.

MOOS, R.H. et R.E. MITCHELL (1982). "Social network resources and adaptation: a conceptual framework», dans T.A. Wills (dir.), Basic processes in helping relationships. New York: Academic Press.

NADLER, A. (1983). "Personal characteristics and help-seeking », dans B.M. DePaulo, A. Nadler et J.D. Fisher (dir.). New directions in helping. Help-seeking, p. 303-340. New York: Academic Press.

OUELLET, F., J. LINDSAY et M.-C. SAINT-JACQUeS (1993). Évaluation d'un programme de traitement pour conjoints violents. Sainte-Foy: Centre de recherche sur les services communautaires, Université Laval.

Piros-GoOd, M. et J. STETS-KEALY (1985). "Male batterers and battering : a national survey», Response, vol. 8: 8-12.

ROBERTSON, J.M. (1989). Men who avoid counseling: identifying personality correlates and preferences for assistance alternatives. Doctoral Dissertation. Santa Barbara: University of California.

RONDEAu, G. (1989). Les programmes québécois d'aide aux conjoints violents : Rapport sur seize organismes existants au Québec. Québec : Ministère de la Santé et des Services sociaux, Direction générale de la planification et de l'évaluation.

ROSEN, S. (1983). "Perceived inadequacy and help-seeking", dans B.M. DePaulo, A. Nadler et J.D. Fisher (dir.). New directions in helping. Help-seeking, p. 73-105. New York: Academic Press.

SARASON, B.R., I.G. SARASON et G.R. PIERCE (1990). "Traditional views of social support and their impact on assessment», dans B.R. Sarason, I.G. Sarason et G.R. Pierce (dir.), Social support : an interactional view, p. 427-453. New York: John Wiley \& Sons.

SAUNDERS, D.G. et J.F. PARKER (1989). "Legal sanctions and treatment follow-through among men who batter: A multivariate analysis», Social Work Research And Abstract, vol. 25, n $3: 21-29$.

SAUNDERS, S.M. (1990). The process of seeking psychotherapy: routes, difficulty and social support. Doctoral Dissertation. Evanston, II.: Northwestern University.

SHAPIRO, G. (1984). "Help-seeking: why people don't», Research in the sociology of organizations, vol. $3: 213-236$.

Sherman, L.W., D.A. SMITH, J. SCHMidT et D.P. Rogan (1992). "Crime, punishment, and stake of conformity: legal and informal control of domestic violence», American Sociological Review, vol. 57 : p. 680-690

SYERS, M. et J.L. EDLESON (1992). "The combined effects of coordinated criminal justice intervention in woman abuse», Journal of interpersonal violence, vol. $7, \mathrm{n}^{\circ} 4: 490-502$.

THOM, B. (1986). "Sex differences in help-seeking for alcohol problems 1. The barriers to help-seeking ", British Journal of Addiction, vol. 81 : 777-788. 
Tijhuis, M.A.R., L. Peters et M. Foets (1990). «An orientation toward help-seeking for emotional problems », Social Science and Medicine, vol. 31, no 9: 989-995.

TOLMAN, R. et L.W. BenNetT (1990). "A Review of Quantitative Research on Men Who Batter», Journal of Interpersonal Violence, vol. 5, $\mathrm{n}^{\circ} 1$ : 87-118.

TOUSIGNANT, M. (1987). L'étiologie sociale en santé mentale. Montréal: Université du Québec à Montréal, Laboratoire de recherche en écologie humaine et sociale.

VAuX, A. (1988). Social support: theory, research, and intervention. New York: Praeger.

WILLIAMS, K.B. et K.C. WILLIAMS (1983). "A social impact perspective on the social inhibition of help-seeking ", dans B.M. DePaulo, A. Nadler et J.D. Fisher (dir.), New directions in helping: Help-seeking, volume 2, p. 187-204. New York: Academic Press.

WILLS, T.A. (1983). "Social comparison in coping and help seeking», dans B.M. DePaulo, A. Nadler et J.D. Fisher (dir.), New directions in helping: Help-seeking, volume 2, p. 109-141. New York: Academic Press.

WILLS, T.A. (1992). "The helping process in the context of personal relationships », dans S. Spacapan et S. Oskamps (dir.), Helping and being helped: naturalistic studies. Newbury Park : Sage. 